\title{
PREVALÊNCIA DE EXCESSO DE PESO EM ESCOLARES DO MUNICIPIO DE REGENTE FEIJÓ-SP E FATORES COMPORTAMENTAIS ASSOCIADOS
}

Edner Fernando Zanuto ${ }^{1 ; 6}$, Gabriel Silva Souza ${ }^{2 ; 6}$, Rômulo Araujo Fernandes ${ }^{3 ; 6}$, Diego Giulliano Destro Christofaro ${ }^{3 ; 6}$, Everton Alex Carvalho Zanuto ${ }^{4 ; 5 ; 6}$

${ }^{1}$ Graduado em Educação Física - UNOESTE, Presidente Prudente - SP. ${ }^{2}$ Discente do Curso Pós Graduação - Latu Senso

- em Fisiologia, Metabolismo do Exercício e Treinamento - UNOESTE, Presidente Prudente - SP. ${ }^{3}$ Docente do Departamento de Educação Física - Presidente Prudente - SP. ${ }^{4}$ Discente do Curso Pós Graduação - Stricto Senso - em Fisioterapia - UNESP, Presidente Prudente - SP. ${ }^{5}$ Docente do Departamento de Educação Física - UNOESTE, Presidente Prudente - SP. ${ }^{6}$ Laboratório de Investigação em Exercício LIVE. Grupo Investigação Científica Relacionada à Atividade Física - GICRAF. Departamento de Educação Física da - UNESP, Presidente Prudente - SP. E-mail: gabrielsilvasolza@hotmail.com.

\section{RESUMO}

O objetivo do presente estudo é investigar a prevalência de excesso de peso e fatores associados em escolares do município em questão. A amostra foi composta por 117 escolares (61 masculinos e 56 femininos) de 6 a 17 anos de idade, matriculados em uma escola municipal de Regente FeijóSP. Foi encontrada uma alta prevalência de excesso de peso (34,5\%). Associação entre sexo, atividade física, comportamento sedentário e IMC entre escolares foi de 0,973;0,443;0,849 respectivamente. A análise não encontrou associação entre as variáveis independentes com o desfecho do estudo. Conclui-se que a população estudada encontra-se com alta prevalência de excesso de peso, juntamente com baixo nível de atividade física e hábitos de vida sedentários.

Palavras-chave: Excesso de peso, crianças, comportamento sedentário, atividade física, sono.

\section{INTRODUÇÃO}

O perfil de doenças no mundo vem apresentando um aumento expressivo nos últimos anos decorrente da alta prevalência de obesidade ${ }^{1,3}$. Nas crianças que se encontram na escola, a predominância de adiposidade corporal e um elevado ganho de peso em espaço curto de tempo são fatores de risco que influenciam para um desenvolvimento de obesidade no futuro ${ }^{4}$.

Estudo feito pela Pesquisa Nacional sobre Demografia e Saúde (PNDS) de 2006 realizado na região Sul do Brasil, constatou uma prevalência nacional de sobrepeso de $6,6 \%$, e de $8,8 \%$ com crianças avaliadas de até cinco anos de idade ${ }^{5}$. A Pesquisa de Orçamento Familiar (POF) de 20082009 revelou uma crescente de 33,5\% na prevalência de excesso de peso no Brasil referente a crianças com idade entre cinco e nove anos, alternando de $32 \%$ a $40 \%$ dentre as regiões Sudeste, Sul e Centro-Oeste, enquanto nas regiões Norte e Nordeste vão de $25 \%$ a $30 \%{ }^{6}$.

Vários estudos vêm demonstrando que a falta de atividade física durante a infância esta relacionada com fatores de risco á saúde durante a idade adulta ${ }^{7,8}$. Outros estudos mostram que menores níveis de atividade física constituem prováveis fatores de risco para a obesidade 
infantil ${ }^{9,10}$. Além disso, foi constatado que o período gasto por crianças pré-escolares em atividades sedentárias (assistir televisão, jogar videogame) esta relacionado a fatores de risco a saúde, assim como sobrepeso e obesidade em idades avançadas da criança ${ }^{11}$.

Não há estudos da comunidade científica que descrevam o nível de obesidade e fatores associados em escolares do município de Regente Feijó, por é de suma importância estudos epidemiológicos, principalmente em países como o Brasil, que estejam em pleno desenvolvimento ${ }^{12}$. Assim sendo, o objetivo do presente estudo é investigar a prevalência de excesso de peso e fatores associados em escolares do município em questão.

\section{MÉTODOS}

\section{Amostra}

O estudo foi submetido ao Comitê de Ética em Pesquisa da Universidade do Oeste Paulista, protocolo numero 1449. Após a sua aprovação todos os responsáveis pelos participantes do estudo assinaram um termo de consentimento livre e esclarecido. A amostra foi composta por 117 crianças e adolescentes de 6-17 anos matriculados em uma escola municipal de Regente Feijó-SP. As avaliações por meio do questionário foram realizadas na própria sala de aula com a supervisão de um auxiliar de pesquisa.

\section{Medidas antropométricas}

O peso foi medido por meio de uma balança eletrônica com precisão de $0,1 \mathrm{~kg}$ e capacidade máxima de $150 \mathrm{~kg}$ Filizola ${ }^{\circledR}$. A estatura foi avaliada por meio de um estadiômetro portátil com precisão de $0,1 \mathrm{~cm}$ e extensão máxima de dois metros. Com a utilização dos valores de massa corporal e estatura foi calculado o Índice de Massa Corporal (IMC).

De posse dos valores de IMC e por meio da utilização dos valores críticos de referência específicos para sexo de acordo com Cole et al. ${ }^{3}$, cada sujeito da amostra foi classificado segundo seu respectivo estado nutricional: eutrofia ou excesso de peso.

\section{Comportamentos sedentários}

Os comportamentos sedentários avaliados foram: número de horas na semana dos escolares assistindo televisão, usando o computador ou jogando videogame. Foram considerados com comportamento sedentário os escolares que reportaram o somatório do uso de televisão, 
videogame e computador igual ou superior a 22 horas semanais, segundo a Academia Americana de Pediatria ${ }^{13}$.

\section{Prática Habitual de Atividade Física}

A prática habitual de atividade física foi obtida por meio do questionário de Baecke et al. ${ }^{14}$, validado para a população brasileira por Florindo et al. ${ }^{15}$. Foram analisados três construtos dessas atividades durante horários de lazer: intensidade (baixa, moderada e vigorosa), tempo semanal de prática (<1h/sem; 1-2h/sem; 2-3h/sem; 3-4h/sem; >4h/sem) e tempo prévio de engajamento $(<1$ mês; 1-3 meses; 4-6 meses; 7-9 meses; >9 meses). Assim, foram considerados fisicamente ativos os indivíduos que relatarem um mínimo de 180 minutos por semana ( $3-4 \mathrm{~h} / \mathrm{sem}$ ) de atividades físicas de intensidade moderada ou vigorosa, nos últimos quatro meses (4-6 meses). Duas categorias grupos foram criadas: (i) indivíduos que relataram menos que 180 minutos por semana / ou intensidade abaixo da estabelecida / ou menor tempo prévio de engajamento; (ii) indivíduos que alcançaram o ponto de corte determinado ${ }^{16,17}$.

\section{Análise Estatística}

Para avaliar a normalidade dos dados obtidos foi utilizado o teste de Shapiro-Wilks, e para associação entre as variáveis o teste do qui-quadrado $\left(X^{2}\right)$ com correção de Yates.

\section{RESULTADOS}

A amostra foi composta por 117 escolares (61 masculinos e 56 femininos) de 6 a 17 anos de idade. Na tabela 1, podemos observar a alta prevalência de excesso de peso $(34,5 \%)$.

Tabela 1. Prevalência de excesso de peso (IMC).

\begin{tabular}{lll}
\hline & Alunos (N) & $\%$ \\
\hline Normal & 76 & 63,9 \\
Excesso de peso & 41 & 34,5 \\
\hline
\end{tabular}

Podemos verificar na tabela 2 , que a análise não encontrou associação entre as variáveis independentes com o desfecho do estudo. 
Tabela 2. Associação entre sexo, atividade física, comportamento sedentário e IMC entre escolares (Regente Feijó-SP 2013).

\begin{tabular}{|c|c|c|c|}
\hline Variáveis Independentes & $\begin{array}{c}\text { Excesso de } \\
\text { Peso } \\
\mathrm{n}(\%)\end{array}$ & $\begin{array}{l}\text { Eutrofia } \\
\qquad \mathrm{n}(\%)\end{array}$ & $\begin{array}{c}\text { Qui-quadrado } \\
\text { p-valor }\end{array}$ \\
\hline \multicolumn{4}{|l|}{ Sexo } \\
\hline Masculino & $19(46,3)$ & $42(55,3)$ & 0,849 \\
\hline Feminino & $22(57,3)$ & $34(44,7)$ & \\
\hline Atividade física & & & 0,443 \\
\hline$<180 \mathrm{~min} / \mathrm{sem}$ & $29(70,7)$ & $60(78,9)$ & \\
\hline$\geq 180 \mathrm{~min} / \mathrm{sem}$ & $12(29,3)$ & $16(21,1)$ & \\
\hline Comportamento Sedentário & & & 0,973 \\
\hline Baixo & $4(9,8)$ & $9(11,8)$ & \\
\hline Elevado & $37(90,2)$ & $67(88,2)$ & \\
\hline
\end{tabular}

\section{DISCUSSÃO}

O objetivo do presente estudo foi investigar a prevalência de excesso de peso e fatores associados em escolares do município em questão.

Este estudo composto por crianças pré-escolares de ambos os sexos, residentes na cidade de Regente Feijó demonstrou uma alta prevalência de excesso de peso entre os participantes $(34,5 \%)$. Alta prevalência que também foi encontrada por Brasil et al. ${ }^{18}$, onde $33,6 \%$ da população de crianças estuda apresentou excesso de peso, sendo $54,5 \%$ a prevalência em escolas privadas e $15,6 \%$ em escolas publicas, não encontrando diferença significativa entre os sexos.

Estudo realizado com crianças portuguesas ${ }^{19}$ mostrou que a realidade de outros países não é muito diferente da nossa, já que foi encontrada uma prevalência de excesso de peso de 14,1\% entre os meninos, enquanto entre as meninas esse valor foi de $18,0 \%$.

Em comparação com meninos $(46,3)$ e meninas $(57,3)$, podemos observar uma maior prevalência de excesso de peso entre as meninas. Característica essa que se assemelha com outros estudos $^{19}$. Contudo, em nossa pesquisa, em relação aos dados entre meninos e meninas não ouve associação $(0,849)$ com o resultado final do mesmo.

Referente a pratica de atividade física, foi notado que 70,7\% das crianças com excesso de peso praticam $<180 \mathrm{~min} / \mathrm{sem}$ de atividade física, enquanto apenas $29,3 \%$ praticam $\geq 180 \mathrm{~min} / \mathrm{sem}$.

Outro achado muito importante foi o alto comportamento sedentário das crianças com excesso de peso $(90,2 \%)$. Dados estes que se assemelham com os achados de Rivera et al. ${ }^{20}$, onde 93,5\% dos jovens avaliados apresentam comportamento sedentário. Em seu estudo, Silva ${ }^{21}$ 
mostrou que $85 \%$ dos meninos e $94 \%$ das meninas apresentavam hábitos de vida sedentários. Já

Oehlschlaeger et al. ${ }^{22}$ demonstraram que $39 \%$ da população estudada era sedentária.

Azambuja et al. $^{23}$, detectaram em seu estudo que crianças que tinham hábitos de assistir acima de 4 horas de TV diariamente estariam 1,4 vezes mais propensos a terem excesso de peso quando comparados as crianças que assistem menos de 4 horas diárias. Afirmaram também que crianças acostumas a comer diante da TV, computador ou videogame, demonstram ser $34 \%$ mais predispostas a ter excesso de peso.

Em seu estudo Olvera et al. ${ }^{24}$, mostram o aumento da atividade física como um efeito protetor para o excesso de peso. Outros estudos também indicaram a pratica da atividade física como um fator positivo na prevenção de excesso de peso ${ }^{24,25}$.

Uma limitação referente ao presente estudo se deve ao tamanho reduzido da amostra estuda, não sendo possível assim, estabelecer um nível expressivo de significância.

\section{CONCLUSÃO}

Assim sendo, com base nos resultados encontrados, pode-se dizer que a prevalência de excesso de peso em escolares encontra se em um nível muito elevado. Conclui-se também que, um percentual grande dessa população encontram se com hábitos sedentários e com nível baixo de atividade física que eleva ainda mais essa prevalência.

\section{REFERÊNCIAS}

1. Obesity: preventing and managing the global epidemic. Report of a WHO consultation. World Health Organ Tech Rep Ser. 2000;894:i-xii, 1-253.

2. Dietz WH, Bellizzi MC. Introduction: the use of body mass index to assess obesity in children. Am J Clin Nutr. 1999;70:123S-5S.

3. Cole TJ, Bellizzi MC, Flegal KM, Dietz WH. Establishing a standard definition for child overweight and obesity worldwide: international survey. BMJ. 2000;320:1240-3. http://dx.doi.org/10.1136/bmj.320.7244.1240

4. Lanigan J, Singhal A. Early nutrition and long-term health: a practical approach. Proc Nutr Soc. 2009;68:422-9. http://dx.doi.org/10.1017/S002966510999019X

5. Brasil. Ministério da Saúde. Pesquisa Nacional de Demografia e Saúde da Criança e da Mulher PNDS 2006: dimensões do processo reprodutivo e da saúde da criança. Brasília: Ministério da Saúde; 2009. 300 
6. Instituto Brasileiro de Geografia e Estatística (IBGE). Pesquisa de orçamentos familiares 20082009: antropometria e estado nutricional de crianças, adolescentes e adultos no Brasil. Rio de Janeiro: IBGE; 2010.

7. Dietz WH. Overweight in childhood and adolescence. N Engl J Med 2004;350(9):855-7. http://dx.doi.org/10.1056/NEJMp048008

8. Craigie AM, Lake AA, Kelly SA, Adamson AJ, Mathers JC. Tracking of obesity--related behaviours from childhood to adulthood: A systematic review. Maturitas 2011;70(3):266-84. http://dx.doi.org/10.1016/j.maturitas.2011.08.005

9. Troiano RP, Flegal KM. Overweight children and adolescents: description, epidemiology, and demographics. Pediatrics 1998;101:497-504.

10. Reilly JJ, Dorosty AR, Emmett PM. Prevalence of overweight and obesity in British children: cohort study. Br Med J 1999;319:1039. http://dx.doi.org/10.1136/bmj.319.7216.1039

11. Moore LL, Gao D, Bradlee ML, Cupples LA, Sundarajan-Ramamurti A, Proctor MH et al. Does early physical activity predict body fat change throughout childhood? Prev Med 2003;37(1):10-27. http://dx.doi.org/10.1016/S0091-7435(03)00048-3

12. Barros SSH, Lopes AS, Barros MVG. Prevalência de baixo nível de atividade física em crianças pré-escolares. Rev Bras Cineantropom Desempenho Hum 2012, 14(4):390-400.

13. American Academy of Pediatrics. Committee on Public Education. American Academy of Pediatrics: Children, adolescents, and television. Pediatrics 2001;107:423-6. http://dx.doi.org/10.1542/peds.107.2.423

14. Baecke JA, Burema J, Frijters JE. A short questionnaire for the measurement of habitual physical activity in epidemiological studies. Am J Clin Nutr 1982; 36: 936-42.

15. Florindo AA, Latorre Mdo R, Jaime PC, Tanaka T, Zerbini CA. Methodology to evaluation the habitual physical activity in men aged 50 years or more. Rev Saude Publica. 2004; 38(2): 307-14. http://dx.doi.org/10.1590/S0034-89102004000200022

16. Fernandes RA, Zanesco A. Early physical activity promotes lower prevalence of chronic diseases in adulthood. Hypertens Res. 2010;33(9):926-31. http://dx.doi.org/10.1038/hr.2010.106

17. Fernandes RA, Christofaro DG, Casonatto J, Codogno JS, Rodrigues EQ, Cardoso ML, Kawaguti SS, Zanesco A. Prevalence of dyslipidemia in individuals physically active during childhood, adolescence and adult age. Arq Bras Cardiol. 2011;97(4):317-23. http://dx.doi.org/10.1590/S0066$\underline{782 \times 2011005000083}$

18. Brasil LMP, Fisberg M, Maranhão HS. Excesso de peso de escolares em região do Nordeste Brasileiro: contraste entre as redes de ensino pública e privada. Rev. Bras. Saúde Matern. Infant., Recife, 7 (4): 405-412, out. / dez., 2007 
19. Silva AJ, Carvalhal MI, Reis VM, Mota MP, Garrido ND, Pitanga F, Marinho D. A prevalência do excesso de peso e da obesidade entre crianças portuguesas. Fit Perf J. 2008 set-out;7(5):301-5.

20. Rivera IR, Silva MAM, Silva RDTA, Oliveira BAV, Carvalho ACC. Atividade Física, Horas de Assistência à TV e Composição Corporal em Crianças e Adolescentes. Arq Bras Cardiol 2010; 95(2) : 159-165. http://dx.doi.org/10.1590/S0066-782X2010005000065

21. Silva RCR, Malina RM. Nível de atividade física em adolescentes do Município de Niterói, Rio de Janeiro, Brasil. Cad Saúde Pública. 2000; 16 (4): 1091-7.

22. Oehlschlaeger MHK, Pinheiro RT, Horta B, Gelatti, San'Tana P. Prevalência e fatores associados ao sedentarismo em adolescentes de área urbana. Rev Saúde Pública. 2004; 38: 157-63. http://dx.doi.org/10.1590/S0034-89102004000200002

23. Azambuja APO, Netto-Oliveira ER, Azambuja MA, Oliveira AAB, Rinaldi W. Prevalência e fatores associados ao excesso de peso em escolares. Revista baiana de saúde pública jul./set. 2012; v.36, n.3, p.740-750.

24. Olvera N, Graham M, McLeod J, Stephanie FK, Butte NF. Prometing moderate-vigorous physical activity in overweight minority girls. Int J Pediatr. 2010; 2010(2010):1-7. doi: 10.1155/2010/415123. http://dx.doi.org/10.1155/2010/415123

25. Farias ES, Paula F, Carvalho WRG, Gonçalves EM, Baldin AD, Guerra-Júnior G. Efeito da atividade física programada sobre a composição corporal em escolares adolescentes. J Pediatr. 2009, 85(1):28-34. doi: 10.2223/JPED.1864. http://dx.doi.org/10.2223/JPED.1864 